\title{
The influence of the protein Wnt10b as a marker of bone repair of critical size defects fille with autogenous adipose tissue graft: A study in rabbit calvaria
}

Luiz Gustavo Klug ${ }^{1}$, Carmen Lucia Mueller Storrer², Aline Monise Sebastiani ${ }^{3}$, Bruna Lorena Barbaresco, Allan Fernando Giovanini ${ }^{2}$, Tatiana Miranda Deliberador ${ }^{2}$

'MSc. Graduate Program in Clinical Dentistry, Positivo University, Curitiba, PR, Brazil

${ }^{2}$ PhD. Professor of the Graduate Program in Clinical Dentistry, Positivo University, Curitiba, PR, Brazil.

${ }^{3} \mathrm{PhD}$ Student in Graduate Program in Clinical Dentistry, Positivo University, Curitiba, PR, Brazil.

${ }^{4}$ Master's student in Graduate Program in Clinical Dentistry, Positivo University, Curitiba, PR, Brazil
Corresponding author: Tatiana Miranda Deliberador Rua Professor Pedro Viriato Parigot de Sousa, 5300 Campo Comprido, CEP: 81280-330, Curitiba/PR, Brasil E-mail: tdeliberador@gmail.com Phone: + 554199764948 Fax: + 554133173403

Received: July 26, 2017 Accepted: October 20, 2017
The proteins Wnts are considered a key regulator of the early development of the skeleton. Aim: The aim of this study was to evaluate the presence of the protein Wnt10b as a marker of bone repair in critical size defects surgically created in the calvaria of rabbits treated with fragmented autogenous adipose tissue graft. Methods: A total of 28 rabbits were divided into two groups: the Control group (C) and Adipose Tissue Graft group (ATG). A CSD measuring $15 \mathrm{~mm}$ in diameter was created in the calvaria of each animal. In rabbits of the $C$ group, the defect was filled only with blood clot, and in ATG group, the defect was filled with fragmented adipose tissue graft. The two groups were divided into two subgroups $(n=7)$ for euthanasia 15 and 40 days after surgery. Histological and immunohistochemically analyses were performed to evaluate the neoformed bone and the presence/concentration of Wnt10b protein. The Kruskal-Wallis test was performed to compare the means and standard deviations of the number of Wnt $10 \mathrm{~b}+$ cells $/ \mathrm{mm}^{2}$ in both groups in each postoperative period. It was assumed a significance level of $5 \%$. Results: After 40 days, the mean concentration of the protein Wnt10b in ATG group was $26.26(+-6.97)$ significant higher $(p<0,001)$ than the mean in $\mathrm{C}$ group that was 305 (37.41). Conclusion: The protein Wnt10b would play a crucial role in the signaling of bone formation in bone defects treated with fragmented autogenous adipose tissue graft.

Keywords: Adipose Tissue. Bone Regeneration. Rabbits. Wnt proteins. 


\section{Introduction}

The growing occurrence of bone disorders and the increase in aging population have resulted in the need for more effective therapies to meet this request. Bone tissue engineering strategies, by combining biomaterials, cells, and signaling factors, are seen as alternatives to conventional bone grafts for repairing or rebuilding bone defects ${ }^{1}$. The successful repair of bone deficiencies still remains a great challenge to surgeons worldwide ${ }^{2}$. Recently, the adipose-derived mesenchymal stem cells have raised a lot of attentions in bone regeneration owing to their easy accessibility and availability in relatively large quantities ${ }^{3}$. Adult stem cells derived from adipose tissue are multipotent cells that can differentiate into osteoblasts in vitro ${ }^{4}$. When these cells are combined with appropriate scaffolds, they can contribute towards bone neoformation in critical size bone defects. In 2011, Zou et al. ${ }^{5}$ have demonstrated that cells derived from adipose tissue have the potential to differentiate into an osteogenic lineage both in vitro and in vivo. The authors stated that this new type of cell-based therapy can be useful for the treatment of bone defects in the clinical field.

To understand bone signaling when adipose tissue graft is used, it is important to know that a signaling mechanism occurs by means of cell markers, such as the Wnt $/ \beta$ catenin. This marker signals the control pathway for the differentiation of various cells because it regulates the expression of target genes. A study in vitro has shown that the $\mathrm{Wnt} / \beta$ catenin signaling pathway inhibited the adipogenic differentiation potential and altered the cell fate from adipocytes to osteoblasts ${ }^{6}$. An in vivo prior study demonstrated that the expression of Wnt 10b in the bone marrow of mice resulted in an increase in the metaphyseal bone volume, which made the bone mechanically stronger?

Wnt signaling pathways are a key regulator of the early development of the skeleton. The activation of the canonical pathway results in the stabilization of $\beta$-catenin. The suppression of Wnt target genes occurs in the absence of $\beta$-catenin. Therefore, $\beta$-catenin can be considered an important molecule that regulates the canonical Wnt signaling pathway to embryogenesis ${ }^{8}$. In a genetic study, the inactivation of $\beta$-catenin, in mesenchymal lineage cells, resulted in severe loss of bone mass due to reduced maturation of osteoblasts as well as increased differentiation of osteoclasts 9 . In addition, an increased Wnt signaling pathway, through a stabilized form of $\beta$-catenin, can produce strengthened ossification as well as the suppression of chondrogenesis 9.10 .

Although the Wnts may inhibit adipocyte differentiation through $\beta$-catenin dependent or independent mechanisms, there is some evidence that the $\mathrm{Wnt} / \beta$-catenin signaling pathway can be considered an important regulator in the adipocyte differentiation ${ }^{11}$.

There are more than 19 proteins and more than 19 receptors and co-receptors, which combine to activate pathways. The proteins $\mathrm{Wnt} 1,2,3,3 \mathrm{~A}, 8 \mathrm{~A}, 8 \mathrm{~B}, 10 \mathrm{~A} \mathrm{E} 10 \mathrm{~b}$ are proven by the canonical Wnt/ $\beta$-catenin; Wnt 4, 5B, 6, 7, 7B and 11 are responsible for activation of the $\mathrm{Wnt} / \mathrm{Ca}^{2+}$ pathway which elevates the cytoplasmic levels of $\mathrm{Ca}^{2+}$ and as Wnt $5 \mathrm{~A}$ and 11 proteins via Planar Cell Polarity (PCP) responsible for the cytoskeletal reorganization ${ }^{12,13}$.

The aim of this study was to evaluate the presence of the protein Wnt10b as a marker of bone repair in critical size defects surgically created in the calvaria of rabbits treated with fragmented autogenous adipose tissue graft. 


\section{Materials and Methods}

This study was approved by the Ethics and Research Committee from Positivo University, Curitiba-PR, Brazil (protocol number: 009/2009). A total of 28 male rabbits Oryctolagus cuniculus (New Zealand strain), aged approximately 6 months and weighing from 2.0 to $3.5 \mathrm{~kg}$ were used. The animals were healthy and had a period of 15 days to adapt themselves to the environment. The rabbits were kept in individual cages at the Positivo University biotery. They were given commercial feed and mineral water ad libitum. The animals were randomly divided into two groups of 14 animals: C group (control) and ATG group (fragmented autogenous adipose tissue graft).

One hour before surgery, rabbits received intramuscular injection of enrofloxacin (0.1 mL / kg) (Baytril, Bayer S.A., São Paulo, SP, Brazil). For the surgical procedure, all the animals received as preanesthetic medication: $1 \mathrm{mg} / \mathrm{kg}$ of midazolam (União Química, São Paulo, SP, Brazil) administered intramuscularly. After sedation, the animals received an anesthetic medication containing a combination of $35 \mathrm{mg} / \mathrm{kg}$ of $10 \%$ ketamine hydrochloride (Syntec, Coria, SP, Brazil) with $5 \mathrm{mg} / \mathrm{kg}$ of $2 \%$ xylazine hydrochloride (Vetbrands, Brazil) intramuscularly. After induction of the anesthetic plan, the venous access was established by the marginal vein of the ear for anesthetic maintenance during the surgical procedure. The rabbit was then positioned on the surgical table in dorsal decubitus position. The anesthesia was maintained by the vaporization of isoflurane (Cristália, Itapira, SP, Brazil) by facial mask.

Critical-size defects (CSD) measuring $15 \mathrm{~mm}$ were created in the calvaria of each animal according to a research methodology described by Oliveira et al. ${ }^{14}$. In $\mathrm{C}$ group, the defect was filled only with blood clot, and in ATG group, the defect was filled with fragmented autogenous adipose tissue graft.

The method used to obtain autogenous adipose tissue was described by Torres et al. [28]. An incision measuring between 2 and $3 \mathrm{~cm}$ was performed with a scalpel blade 15 , about $5 \mathrm{~cm}$ posterior to the skull base, along the dorsomedial line, towards the craniocaudal direction. The adipose tissue was carefully removed from the exposed area with sterile scissors and tweezers (Figure 1). Then, it was macerated with a scalpel blade 15, through random cuts in different directions, and immediately placed in the surgical defect.

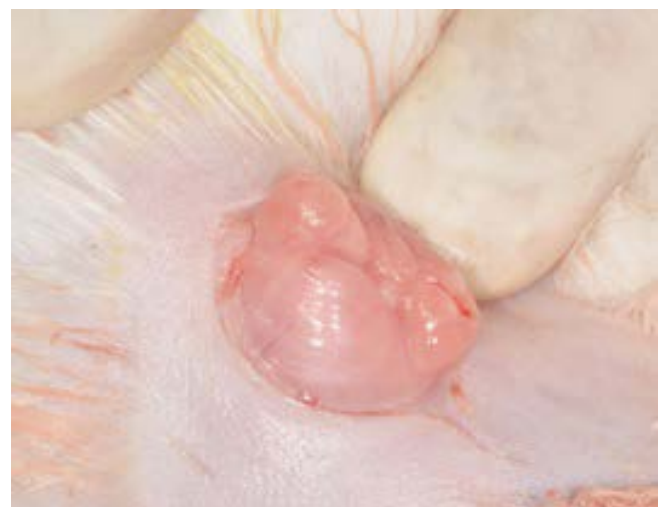

Figure 1. Incision and exposure for the removal of adipose tissue from animal's dorsal region. 
The soft tissues were then repositioned and sutured for a primary closure of the wound. The suture was performed in two layers. In the first layer, the periosteum was sutured with absorbable suture thread (4-0 Medcryl, MedEvolution, Tamil Nadu, India). In the second layer, dermis and epidermis were sutured with 4-0 silk suture thread (Ethicon, Johnson \& Johnson, São José dos Campos, SP, Brazil).

Each animal was given intramuscular injections of enrofloxacin ( $5 \mathrm{ml}$ per $\mathrm{kg}$ of body weight) once a day for five days. To control postoperative pain, the animals were given morphine ( $3 \mathrm{mg} / \mathrm{kg}$ intramuscular) at the end of the surgery and 4 hours after the first injection. Analgesia was maintained with paracetamol dissolved in water $(200 \mathrm{mg} / \mathrm{kg})$ 3 times a day for 5 days.

The $C$ and ATG groups were divided into two subgroups with 7 animals for euthanasia in 15 and 40 days' time after surgery. The euthanasia was performed through a fast intravenous injection of Thiopental Sodium 2.5\% (10 ml/ $\mathrm{kg})$.

\section{Tissue Processing}

The original surgical defect area and its surrounding tissue were removed in blocks. The specimens were fixed in 10\% neutral formalin, washed in tap water, and decalcified in $20 \%$ formic acid. After decalcification, each specimen was hemi-sectioned in its core, parallel to the sagittal suture. The specimens were processed and embedded in paraffin. Longitudinal serial sections, $3 \mu \mathrm{m}$ thick each, were performed, starting from the core of the original surgical defect. The sections were stained with the technique of Hematoxylin and Eosin (HE) for posterior analyses with light microscopy.

\section{Histological Analysis}

Two histological sections were selected from each animal, representing the core of the original surgical defect. These analyses were performed by a single operator previously calibrated.

For histological analysis, the images were analyzed in $12 x$ and $40 x$ magnification by means of an optical microscope (021/3 Quimis, Diadema, SP, Brazil) and the following parameters were assessed: the closure of the bone defect, the amount of the newly bone matrix deposition, a quantity of presence of dense connective tissue, the presence of acute (neutrophylic) or chronic (mononuclear) inflammatory infiltrate. The closure of the defect was considered complete when all its extensiveness was filled with newly formed bone. Each parameter was considered such as negative $(-)$, scarce $(+)$, moderated (++) and intense (+++).

\section{Immunohistochemical analysis}

The Immunohistochemical antiperoxidase was performed with the use of the protein Wnt 10b. The immunoexpression of the protein was obtained through the method streptavidin-biotin immunoperoxidase. Three-micrometer-thick cuts of each specimen were deparaffinised and submitted to antigen retrieval in $1 \%$ trypsin solution ( $\mathrm{pH}$ 6.8) for 60 minutes at 37-C for the anti-Wnt 10b and $10 \mathrm{mM}$ citrate solution (pH 6.0) for 45 minutes in a double-boiler at 95-C for the anti-CD34 antibody. The slides containing the histologic specimens were immersed in $3 \%$ hydrogen peroxide for 30 minutes 
to extinguish endogenous peroxidase activity, followed by incubation with $1 \%$ phosphate-buffered saline ( $\mathrm{pH}$ 7.4). The sections were incubated with the primary antibody anti-Wnt $10 \mathrm{~b}(0.5 \mathrm{mg} / \mathrm{mL}$, ab 6285; Abcam, Cambridge, UK) with a dilution factor of 1:100. A labelled streptavidin biotin antibodybinding detection system (Universal HRP Immunostaining Kit; Diagnostic Biosystem, Foster City, CA) was used to detect primary antibodies. The immune reaction was revealed with diaminobenzidine tetrachloride chromogen solution (Diagnostic Biosystem), which produced a brown precipitate at the antigen site. The specimens were counterstained with Mayer hematoxylin. A negative control was performed for all samples using rabbit polyclonal isotype IgG $(2 \mathrm{Kg} / \mathrm{mL}$, ab 27472, Abcam) for 10 minutes at room temperature as a primary antibody. For each specimen, 3 slides were used for incubation with each antibody.

The images of each field were obtained with a light microscope with a magnification of X 400 through bright-field fixed focus. Then, the images displayed on a TV monitor connected to a computerized system were scanned by a system of cameras (Samsung SDC-310, Korea) attached to a light microscope (021/3 Quimis, Diadema, SP, Brazil). After that, a manual counting of the nuclei that expressed immunostaining of the protein was performed.

The immunopositive cells for the antibody Wnt 10b were counted twice in every surgical defect by a single observer. For the statistical analysis of the immunohistochemical expression of the antigen investigated, the results were expressed as a percentage, taking into account the relation between the number of positive cells and the number of total cells showing mesenchymal morphology.

\section{Statistical Analysis}

The data were submitted to descriptive and inferential statistical analysis. The non-parametric statistical test of Kruskal-Wallis was performed to compare the mean and standard deviations of the number of Wnt $10 \mathrm{~b}+$ cells $/ \mathrm{mm}^{2}$ between groups. Was assumed a significance level of $5 \%$. All histological parameters were analyzed by Moda.

\section{Results}

\section{Histological Analysis}

The histometric aspects found in the present study is revealed in the table1, the qualitative results is summarized bellow. No amount of newly formed bone was observed.

Table 1. Histometric aspects found in histological analysis

\begin{tabular}{llccc}
\hline \multirow{2}{*}{ Parameter analyzed } & \multicolumn{2}{c}{15 days } & \multicolumn{2}{c}{40 days } \\
\cline { 2 - 5 } & C & ATG & C & ATG \\
\hline Closure of the bone defect & - & - & - & + \\
\hline Acute (neutrophylic) inflammatory infiltrate & - & - & - & - \\
\hline Chronic (mononuclear) inflammatory infiltrate & + & - & - & - \\
\hline Presence of connective tissue & +++ & +++ & ++ & + \\
\hline Bone matrix & - & - & + & +++ \\
\hline
\end{tabular}




\section{Group Control (C) - 15 days after surgery:}

There was the presence of chronic inflammatory infiltrate amidst thin dense connective tissue whose fibers were arranged parallel in the anteroposterior direction (Figure 2-A). No amount of newly formed bone was observed.

\section{Group Control (C) - 40 days after surgery:}

Only scarce osteoid matrix were identified mainly on surgical defect, but there was one case in which the bone formation occurred in the core areas of the defect as well (Figure 2-C). The defect was composed by intense dense connective tissue.

\section{Group Fragmented Adipose Tissue Graft (ATG) - 15 days after surgery:}

A complete bone closure of the surgical defect was not observed in this period. The reparative histological analysis showed the presence of dense connective tissue with

Control group

15 days

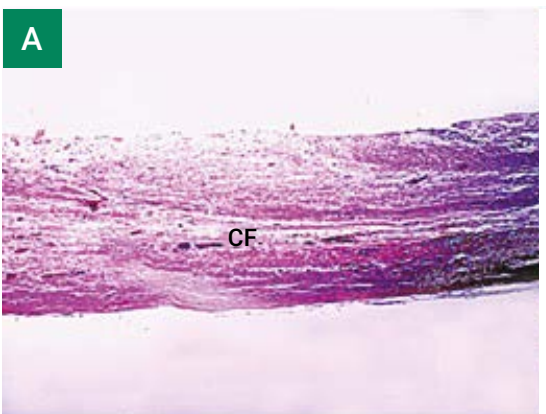

40 days
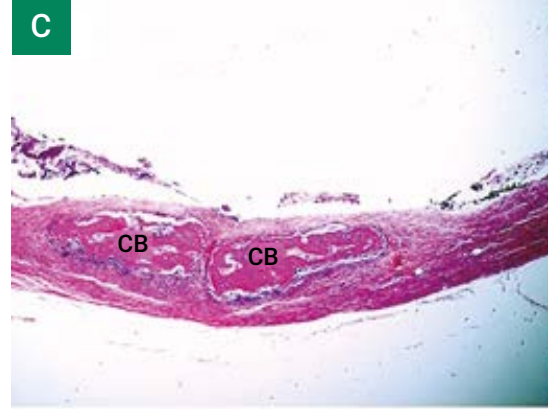

Adipose tissue graft group
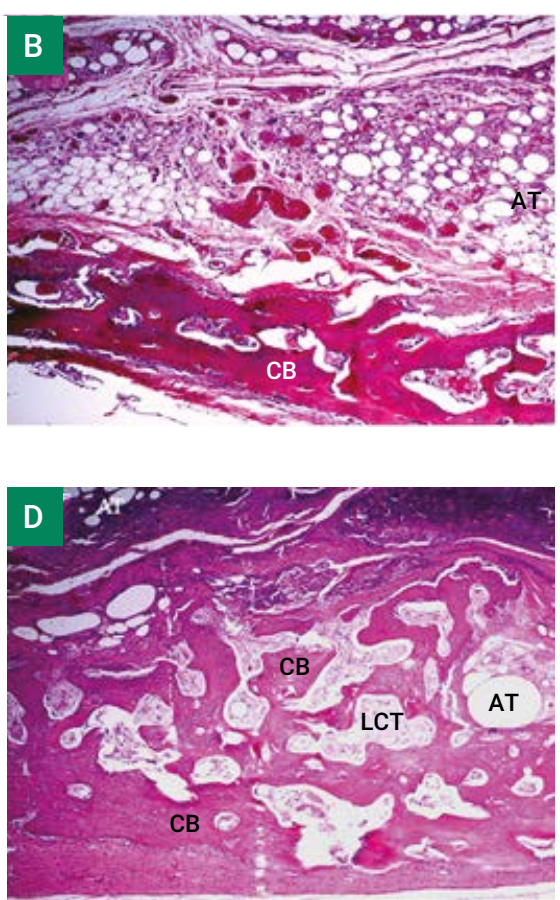

Figure 2. A) Microscopic view of C Group $C$ in the postoperative period of 15 days. This view shows an intense deposition of collagen fibers (CF) parallel to the defect. (HE staining, original magnification $\times 40$ ). B) Microscopic aspect of ATG Group in the postoperative period of 15 days. Microscopy reveals remaining adipose tissue (AT) surrounded by osteoid tissue and intense deposition of newly formed compact bone (CB) tissue encircling the defect area. (HE staining, original magnification $\times 40$ in). C) Microscopic view of Group $C$ in the postoperative period of 40 days. There was neoformation of compact bone (CB) mimicking areas of intramembranous formation during this period. A large area of the defect was predominantly filled with dense connective tissue. (HE staining, original magnification × 40). D) Microscopic aspect of Group ATG in the postoperative period of 40 days. The microscopic view shows intense deposition of mature compact bone (CB) tissue filling a large part of the artificial defect. Most adipose tissue (AT) was replaced by bone tissue or loose connective tissue (LCT) mimicking the bone marrow extracellular matrix. (HE staining, original magnification $\times 40$ ). 
bundles of collagen fibers amidst the remaining adipose tissue, sometimes encircling the tissue. These fibers also had a pale coloring that characterizes the formation of osteoid structure, in which isolated fragments of mature newly formed tissue were found. Newly formed bone tissue was evident in the margins of the defect. The tissue thickness was similar to the original bone volume of the skullcap (Figure 2-B).

Group Fragmented Adipose Tissue Graft (ATG) - 40 days after surgery:

The results were heterogeneous among the specimens. Some specimens had a larger amount of osteoid tissue while others had areas filled with newly formed compact Haversian bone tissue, not only surrounding the defects but also amidst the bundles of collagen fibers. Discrete adipose tissue remaining in the center of the defect could be seen as well (Figure 2-C).

Immunohistochemical analysis of the protein WNT 10b

All specimens showed the presence of Wnt10b + cells. The ATG group showed intense positivity to cells $/ \mathrm{mm}^{2}$ in areas with dense connective tissue, osteoid tissue, and con-

Control group

15 days

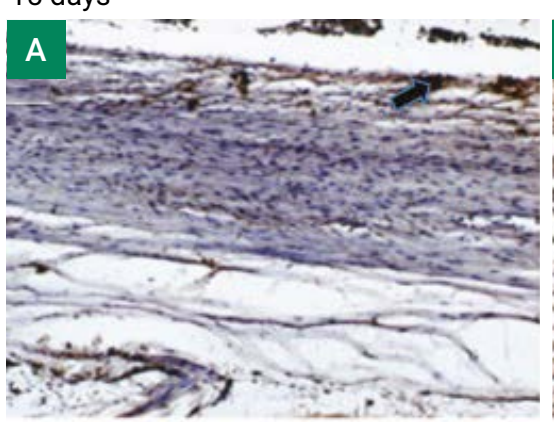

40 days

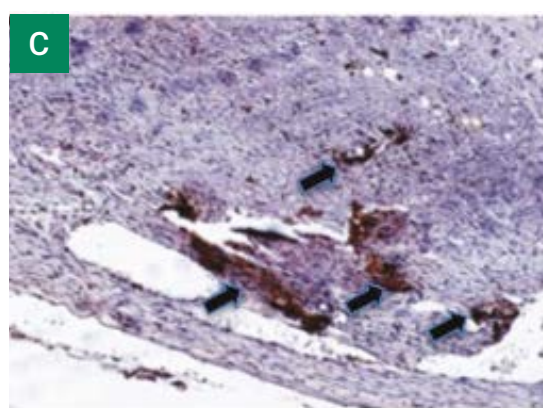

Adipose tissue graft group
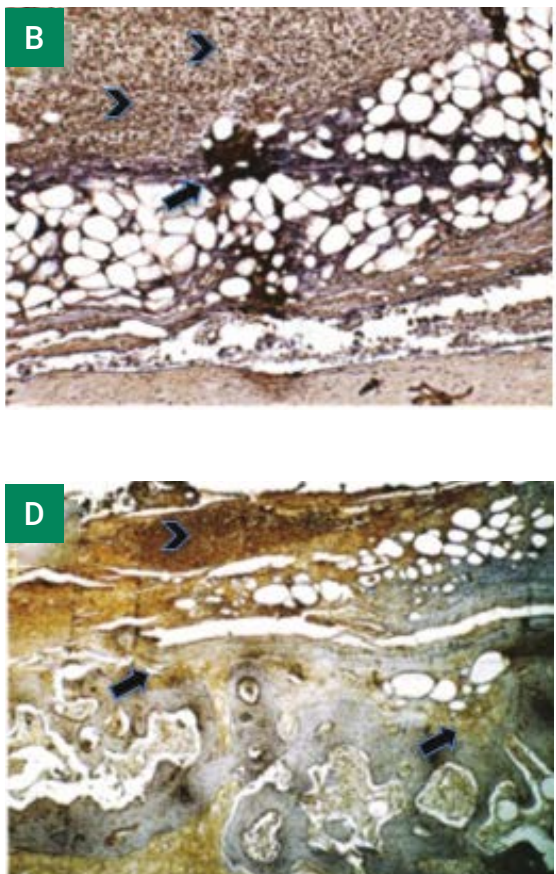

Figure 3. A) Immunohistochemical aspects of Control group in the postoperative period of 15 days. Verify the presence of Wnt10b localized on specific stromal area (arrows) B) Immunohistochemical aspects of ATG group in the postoperative of 15 days. There are important presence of Wnt $10 \mathrm{~b}$ on nucleus of cells spread on fibrous tissue (head of arrow) and immunopresence of Wnt10b in mineralization areas surrounding the adipose tissue (arrows) E) Immunohistochemical aspects of Control group in the postoperative of 40 days. It shows intense presence of dense connective tissue surrounding scarce area of osteoid Wnt10b + (arrows) F) Immunohistochemical aspects of ATG group in the postoperative of 40 days (Original magnification $\times 40$ ). Verify the intense presence of Wnt $10 b+$ cells peripherally to bone matrix deposition on deffects (arrows) and also in the conective tissue (head of arrows). 
Table 2. Means and standard deviations of the number of Wnt $10 / \beta+$ cells $/ \mathrm{mm}^{2}$ in both groups in each postoperative period.

\begin{tabular}{lcc}
\hline \multirow{2}{*}{ Postoperative Period } & \multicolumn{3}{c}{ GROUPS } \\
\cline { 2 - 3 } & Group C & Group ATG \\
\hline 15 days & $71.93 \pm 22.28 \mathrm{bcd}$ & $189.08 \pm 28.36 \mathrm{ab}$ \\
\hline 40 days & $26.23 \pm 6.97 \mathrm{~d}$ & $305.33 \pm 37.41 \mathrm{a}$ \\
\hline
\end{tabular}

According to the non-parametric statistical test of Kruskal-Wallis, the number followed by the same letter shows statistical similarities $(\mathrm{p}<0.05)$

nective tissue septa. In C group, a greater amount of positive cells was found in the edges of the defect.

In C group, the amount of positive cells $/ \mathrm{mm}^{2}$ remained regular in both periods, reaching an immunostaining peak in the postoperative period of 15 days (Figure 3-A). In the period of 40 days, these values decreased coinciding with the deposition of bone neoformation (3-C). The ATG group showed in postoperative of 15 days, intense positivity in the formation of osteoid tissue among the adipose cells (notched arrows) and positivity of fat cells to the protein analyzed, suggesting genetic reprogramming of mature cells. In this group, there was a crescent number of cells that showed positivity to Wnt10b in relation to the period of time analyzed, even when the bone matrix was deposited (Figure 3-B,D). The table 02 compare the means of Wnt10b proteins concentrations in the groups.

\section{Discussion}

Bone repair is a complex biological process characterized by controlled bone recovery, through osteogenesis and osteoinduction, so that the functional and structural characteristics of injured tissue can be restored in the areas where there is a bone defect ${ }^{15}$. We chose to perform a critical size defect of $15 \mathrm{~mm}$ according to the study of Maiti in $2016^{16}$, because we believe it is a defect size sufficient to hinder bone formation by the normal cicatrization process from the clot. Allowing the comparison of different levels of bone formation to correlate with the presence of the Wnt10b protein

An ideal grafting material for bone repair is still a big challenge, and object of various researches. Adipose tissue has proven to be rich in adult stem cells, and as a result, it has been the focus of extensive research on the regeneration of lost tissue ${ }^{17}$. Therapies based on stem cells for the repair and regeneration of different kinds of tissue and organs have been considered, at least in theory, a therapeutic alternative with satisfactory solutions for a great number of diseases. Stem cells have been widely used in experimental trials in order to develop new techniques for the repair of bone tissue ${ }^{18}$.

In this regard, the autogenous adipose tissue can theoretically serve as an important and abundant source of multipotent adult stem cells, since it contains approximately 100 times more stem cells than the bone marrow do, with suitable properties for tissue engineering and tissue regeneration with orthopedic purposes ${ }^{19}$. Moreover, new findings show that subcutaneous adipose tissue and bone marrow tissue share the same stem cell phenotype. Studies have demonstrated the intimate relation between these two types of tissue (since bone and fat have the same cell clone). In addition, adipose tissue cannot only be genetically reprogrammed but can also transdifferentiate into bone tissue ${ }^{20}$. 
Our data suggest that the subcutaneous fat tissue graft could be a plausible osteoconductive biomaterial. The outcome has shown that the use of fat tissue increased the volume of tissue inside the bone bed providing a satisfactory bone formation. However, there is a need for new studies with histomorphometric analysis comparing fat graft with autogenous graft and with bone substitutes.

When adipose tissue grafting was used (Group ATG), the histological results showed moderate bone deposition in the peripheral regions of the defect delimiting periosteal and endosteal areas, mimicking a 'sandwich' of intramembranous bone formation, and trabecular formation, with areas of de neovascularization as well as a reorganization of adipose tissue in bone graft areas. Later, a higher bone density (in the period of 40 days) and substantial loss of grafted adipose tissue could be observed. Thus, there were two independent, distinct processes of bone neoformation in Group ATG and Group C. In Group C, the bone neoformation was achieved more slowly and more concentrated around the remaining bone area (the edges of the defect), where there were medullar fragments and periosteum. The ossification process occurred in the regions where there was a higher concentration of cells marked by Wnt10b.

Nowadays, it is well-known that regulatory factors that induce and control the development of skeletal structures arise from regulatory factors that include the proteins Wnts and all cascades of events produced by their presence ${ }^{9}$. In this work, mineralization and bone tissue formation occurred primarily in the regions of fibrous matrix associated with fusiform-shaped cells, which also comprised the Wnt10b protein.

The expression of this protein was identified by Bennett et al. ${ }^{7}$ in cell lineages inside the bone marrow of mice during a process of bone remodeling. The authors identified a direct correlation between the presence of the protein and the growth of the metafisial bone volume. Furthermore, the authors also identified that the bone that had larger amounts of Wn10b was characterized by a mechanically stronger bone tissue ${ }^{21}$.

A study in vitro has shown that the Wnt/ $\beta$-catenin signaling pathway inhibited the adipogenic differentiation potential and altered the cell fate, from adipocytes to osteoblasts. The activation of the $\mathrm{Wnt} / \beta$-catenin signaled a cell fate change, from adipose-tissue derived mesenchymal stem cells to osteoblastogenesis, at cost to adipogenesis. Our results presented in this study showed, in the initial periods, higher amounts of this protein concentrated in cells that were lodged in areas with connective tissue adjacent to grafted adipose tissue. Later, this protein was also expressed in mature adipose tissue, which decreased in volume while the bone matrix increased. Therefore, the expression of Wnt10b appears to be an important path towards osteogenic differentiation when adipose tissue is grafted onto a craniofacial bone site.

This result suggests a hypothesy of mineralization through cell transdifferentiation ${ }^{22}$. The term transdifferentiation refers to a phenotypic change from one kind of differentiated cell to another kind that is functionally different in terms of lineage. Studies in vitro have demonstrated that such transdifferentiation occurs mainly between adipocytes and osteoblasts due to growth and hormone factors, similarly to the effect of osteoporosis, or "pseudo-osteoporosis"23.

Studies conducted by Gimble and Guilak ${ }^{22}$ have demonstrated that the transdifferentiation phenomenon has important therapeutic implications, though it should be 
regarded with caution since a prior commitment to differentiation, from one or another particular lineage, is a random event. However, this random aspect seems not to exist when subcutaneous adipose tissue is grafted onto rabbit calvaria, since the increase in bone tissue and in osteoid tissue was also associated with the increase in Wnt10b.

The concentration of the protein Wnt10b in ATG group was significant higher, comparing with $\mathrm{C}$ group, suggesting that this protein could play a role in the signaling of bone formation in bone defects treated with fragmented autogenous adipose tissue graft. New studies with measurement of newly bone formed area to compare with histological and immuhistochemical data should be perform to comprove this hypothese.

\section{References}

1. Orciani M, Fini M, Di Primio R, Mattioli-Belmonte M. Biofabrication and Bone Tissue Regeneration: Cell Source, Approaches, and Challenges. Front Bioeng Biotechnol. 2017 Mar 23;5:17. doi: 10.3389/fbioe.2017.00017

2. Xie Q, Wei W, Ruan J, Ding Y, Zhuang A, Bi X, et al. Effects of miR-146a on the osteogenesis of adipose-derived mesenchymal stem cells and bone regeneration. Sci Rep. 2017 Feb 16;7:42840. doi: $10.1038 /$ srep42840.

3. Zuk PA. Human Adipose Tissue Is a Source of Multipotent Stem Cells. Mol Biol Cell. 2002 Dec;13(12):4279-95.

4. Im GI, Shin YW, Lee KB. Do adipose tissue-derived mesenchymal stem cells have the same osteogenic and chondrogenic potential as bone marrow derived cells? Osteoarthritis Cartilage. 2005 Oct;13(10):845-53.

5. Zou J, Wang G, Geng D, Zhu X, Gan M, Yang H. A Novel Cell-Based Therapy in Segmental Bone Defect: Using Adipose Derived Stromal Cells. J Surg Res. 2011 Jun 1;168(1):76-81. doi: 10.1016/j.jss.2009.07.021.

6. Li HX, Luo X, Liu RX, Yang YJ, Yang GS. Roles of Wnt/-catenin signaling inadipogenic differentiation potential of adipose-derived mesenchymal stem cells. Mol Cell Endocrinol. 2008 Sep 10;291(1-2):116-24. doi: 10.1016/j.mce.2008.05.005

7. Bennett CN, Longo KA, Wright WS, Suva LJ, Lane TF, Hankenson KD, et al. Regulation of osteoblastogenesis and bone mass by Wnt10b. Proc Natl Acad Sci U S A. 2005 Mar 1;102(9):3324-9.

8. Logan CY, Nusse R. The Wnt signaling pathway in development and disease. Annu Rev Cell Dev Biol. 2004;20:781-810

9. Glass DA, Bialek P, Ahn JD, Starbuck M, Patel MS, Clevers H, et al. Canonical wnt signaling in differentiated osteoblasts controls osteoclast differentiation. Dev Cell. 2005 May;8(5):751-64..

10. Hill TP, Spater D, Taketo MM, Birchmeier W, C. Hartmann C. Canonical Wnt/beta-catenin signaling prevents osteoblasts from differentiating into chondrocytes. Dev Cell. 2005 May;8(5):727-38.

11. Prestwich TC, Macdougald OA. Wnt/ $\beta$-catenin signaling in adipogenesis and metabolism. Curr Opin Cell Biol. 2007 Dec;19(6):612-7.

12. Endo Y, Wolf V, Muraiso K, Kamijo K, Soon L, Uren A, et al. Wnt-3a-dependent cell motility involves RhoA activation and is specifically regulated by dishevelled-2. J Biol Chem. 2005 Jan 7;280(1):777-86

13. Niehrs C. The complex world of WNT receptor signalling. Nat Rev Mol Cell Biol. 2012 Dec;13(12):767-79. doi: 10.1038/nrm3470

14. Oliveira LD, Giovanini AF, Abuabara A, Klug LG, Gonzaga CC, Zielak JC, et al. Fragmented adipose tissue graft for bone healing: histological and histometric study in rabbits' calvaria. Med Oral Patol Oral Cir Bucal. 2013 May 1;18(3):e510-5 
15. Leucht P, Jiang J, Cheng D, Liu B, Dhamdhere G, Fang MY, et al. Wnt3a reestablishes osteogenic capacity to bone grafts from aged animals. J Bone Joint Surg Am. 2013 Jul 17;95(14):1278-88. doi: 10.2106/JBJS.L.01502

16. Maiti SK, Ninu AR, Sangeetha P, Mathew DD, Tamilmahan P, Kritaniya D, et al. Mesenchymal stem cells-seeded bio-ceramic construct for bone regeneration in large critical-size bone defect in rabbit. J Stem Cells Regen Med. 2016 Nov 29;12(2):87-99.

17. Sterodimas A, de Faria J, Nicaretta B, Pitanguy I. Tissue engineering with adipose-derived stem cells (ADSCs): current and future applications. J Plast Reconstr Aesthet Surg. 2010 Nov;63(11):1886-92. doi: 10.1016/j.bjps.2009.10.028.

18. Trofin EA, Monsarrat P, Kémoun P. Cell therapy of periodontium: from animal to human? Front Physiol. 2013 Nov 15;4:325. doi: 10.3389/fphys.2013.00325.

19. Gomes SP, Deliberador TM, Gonzaga CC, Klug LG, Oliveira LC, Urban CD, et al. Bone healing in criticalsize defects treated with immediate transplant of fragmented autogenous white adipose tissue. J Craniofac Surg. 2012 Sep;23(5):1239-44.

20. Gomillion CT, Burg KJL. Stem cells and adipose tissue engineering. Biomaterials. 2006 Dec;27(36):6052-63.

21. Longo KA, Wright WS, Kang S, Gerin I, Chiang SH, Lucas PC, et al. Wnt10b inhibits development of white and brown adipose tissues. J Biol Chem. 2004 Aug 20;279(34):35503-9.

22. Gimble J, Guilak F. Adipose-derived adult stem cells: isolation, characterization, and differentiation potential. Cytotherapy. 2003;5(5):362-9.

23. Song L, Tuan RS. Transdifferentiation potential of human mesenchymal stem cells derived from bone marrow. FASEB J. 2004 Jun;18(9):980-2. 